\title{
EVALUACIÓN DE LA EFECTIVIDAD DE UN PROGRAMA EDUCA- TIVO EN HIGIENE ORAL DIRIGIDO A CUIDADORES DE ADULTOS MAYORES DE LOS ASILOS SAN ANTONIO Y SAN RAFAEL DE LA CIUDAD DE BUCARAMANGA
}

${ }^{1}$ Yenny Maritza García T., ${ }^{1}$ Luswin J. Flórez M., ${ }^{1}$ Lina María Silva B., ${ }^{2}$ Exiomara Aguilar C. ¿Sonia Constanza Concha $S$

${ }^{1}$ Estudiante de X semestre, F. de Odontología, U. Santo Tomás, ${ }^{2}$ Odontólogo, U. Santo Tomás, Especialista en Educación y Comunicación para la Salud, Magíster en Epidemiología, U. Industrial de Santander, Docente U. Santo Tomás

Autor responsable de correspondencia: Luswin J. Flórez M. Correo electrónico: lupinfmt@ hotmail.com

\section{RESUMEN}

Objetivo: Diseñar, aplicar y evaluar un programa educativo en higiene oral dirigido a los cuidadores de adultos mayores de los asilos San Antonio y San Rafael de la ciudad de Bucaramanga

Materiales y métodos: Se realizó un ensayo clínico no controlado. La muestra estuvo integrada por 25 cuidadores y 94 adultos mayores vinculados a estas dos instituciones durante los años 2004 y 2005. En cuidadores se evaluaron variables sociodemográficas y sus conocimientos y percepciones sobre salud general y oral de los adultos mayores. Para adultos mayores se recolectaron además de las variables sociodemográficas, las relacionadas con nivel de higiene oral de superficies dentales mediante el índice cuantificado de placa (ICP), el índice de cobertura de lengua y el índice de evaluación de higiene de prótesis dentales (IEHP). Las variables sociodemográficas y clínicas recolectadas en adultos mayores se compararon según institución intervenida o no intervenida mediante test Chi Cuadrado y test exacto de Fisher. Se consideró un nivel de significancia $\alpha=0.05$.

Resultados: En el grupo control no hubo diferencias estadísticamente significativas entre el ante y el después de la intervención, contrario al grupo al que se le aplicó el programa educativo.

Conclusiones: Se consideró que intervenir a los cuidadores de adultos mayores institucionalizados, con un programa educativo continuo e integral sobre higiene oral, permite multiplicar y mejorar los niveles de higiene oral en los adultos mayores, comparado una acción puntual dirigida a esta misma audiencia. [García Y, Flores L, Silva L, Aguila E, Concha S. Evaluación de la efectividad de un programa educativo en higiene oral dirigido a cuidadores de adultos mayores de los asilos San Antonio y San Rafael de la ciudad de Bucaramanga. Ustasalud Odontología 2006; 5: 40 - 48]

Palabras clave: Adulto mayor, Institucionalización, Programa educativo, Higiene oral, Cuidadores.

\section{EVALUATION OF THE EFFECTIVENESS OF AN EDUCATIVE PROGRAM IN ORAL HYIGIENE DIRECTED TO CARETAKERS OF THE ELDERLY PEOPLE FROM TWO ASYLUMS OF THE CITY OF BUCARAMANGA}

\begin{abstract}
Purpose: To design, implement and evaluate an educative program in oral hygiene oriented to the caretakers of the elderly of the asylums San Antonio and San Rafael from the city of Bucaramanga.

Material and methods: A not controlled clinical test was made, selecting by convenience the institution taken part with an educative program; the population was represented by 30 geriatrical caretakers and 105 elderly people resident of these two asylums in the years $2004-2005$, that accepted to participate in the investigation. For the evaluation of categorical variables it was made by means of the statistical tests, square Chi test and exact test of Fisher; whereas to the measured quantitative variables in reason scale test $t$ was applied to them of student and/or nonparametric statistical tests according to its nature and distribution.

Results: In the control group, there were no statistically significant associations between before and after the precise intervention, in opposition in the group in which the educative program was applied.

Conclusions: It is considered that to take part to the caretakers of institutionalized elderly people, with an educative program in sequential and integral form on oral hygiene, it allows to multiply as well and, to create healthful conditions in the greater adults, than they stimulate them to implement care mechanisms.
\end{abstract}

Key words: Elderly people, Institutionalization, Educative program, Oral hygiene, Caretakers. 


\section{INTRODUCCIÓN}

Los avances en el área de la salud, pero principalmente el desarrollo de programas preventivos, ha dado como resultado un incremento significativo de la esperanza de vida del ser humano, pues la expectativa de vida que hace 2000 años estaba entre 20 y 30 años, hoy se encuentra en promedio en el mundo entre los 64 y 70 años para los hombres y los 70 y 78 para las mujeres. En Colombia, se estima que para el año 2025 existirán siete millones de personas adultas mayores, lo que equivaldrá al trece por ciento del total de la población adulta. ${ }^{1}$

El envejecimiento se puede considerar como una etapa más del desarrollo evolutivo del ser humano; en la que convergen además de los fenómenos involutivos de disgregación fisiológica y morfológica (que tienden a comprometer la coherencia funcional del organismo) ${ }^{2}$ la modificación de las relaciones sociales, laborales, familiares y de pareja; ${ }^{3}$ lo que contribuye al desarrollo de manifestaciones psicosomáticas a nivel general y oral en el adulto mayor.

A nivel bucodental las alteraciones más frecuentes en esta población son: caries, periodontitis, gingivitis, pérdida de la calidad funcional de las dentaduras, ${ }^{2,46}$ que se relacionan directamente con deficiencias en el estado nutricional, $, 3,8$ lo que afecta la calidad de vida y el bienestar de los adultos mayores.

Cuando éstos y otros aspectos como las condiciones socioeconómicas desfavorables, la ausencia o marginación del grupo familiar y el aumento de las necesidades de asistencia especializada en salud por parte de los adultos mayores, ${ }^{9,10}$ confluyen con el proceso normal de envejecimiento de los individuos, hacen del adulto mayor una persona vulnerable; que requiere de cuidados inherentes a su condición, especialmenteen aquellos que presentan dependencia parcial o total; ${ }^{11}$ por lo que la idea de institucionalizar al adulto se considera una solución adecuada y segura. ${ }^{7,12,13}$

Los centros geriátricos se constituyen, en las alternativas que permiten una mejor atención del adulto dependiente o frágil, por lo tanto, deben contar con un grupo interdisciplinario de cuidadores, con la finalidad de brindar una atención integral al adulto mayor. Sin embargo, estudios previos han mostrado que los cuidadores geriátricos, no tienen los conocimientos suficientes ni una buena actitud ante el cuidado de la salud oral del adulto mayor. ${ }^{1,3,14,15}$ Por lo anterior y por la poca evidencia encontrada en la literatura acerca de estos programas, el propósito de este estudio fueel diseño, aplicación y evaluación de un programa educativo en higiene oral dirigido a los cuidadores de los asilos San Antonio y San Rafael de la ciudad de Bucaramanga.

\section{MATERIALES Y MÉTODOS}

Se realizó un ensayo clínico no controlado. El universo estuvo conformado por 30 cuidadores geriátricos y 236 adultos mayores residentes de los asilos San Antonio y San Rafael de la ciudad de Bucaramanga, durante el segundo semestre del año 2004 y el primer semestre del año 2005. Se excluyeron del estudio aquellos cuidadores y adultos mayores que rehusaron vincularsea la investigación, al igual que aquellos adultos mayores con diagnóstico de patologías psiquiátricas mayores, alteraciones visuales severas y con enfermedades que dificultaran la realización de sus labores de autocuidado.

El tamaño de la muestra para adultos mayores fue calculado mediante rutina simple-size para estudios de cohorte, del programa EPI-INFO v.6.04, ${ }^{16}$ con base en las siguientes especificaciones de diseño: prevalencia de mala higiene oral en el grupo control del $50 \%$ y en el grupo intervenido $20 \%$, definidos en la prueba piloto; OR (Odds Ratio) 0.25 y RR (Riesgo Relativo) 0.4, considerando un nivel de confianza de $95 \%$, un poder del $80 \%$ y una relación de expuesto-no expuesto de 1:1, lo que permitió estimar que una muestra de 90 individuos pertenecientes al grupo control y grupo intervención permitirían detectar diferencias estadísticamente significativas. La muestra de adultos mayores se seleccionó mediante un muestreo no probabilístico por conveniencia; para el caso de los cuidadores todas entrarían en el estudio.

En esta investigación se aplicó un instrumento para adultos mayores, tipo encuesta diligenciado por un evaluador previamente entrenado, en el que se recopilaron variables sociodemográficas como edad, género, nivel educativo y económico, y datos relacionados con su salud general ehigiene oral; para la que se utilizaron los índices: cuantificado de placa (ICP) para evaluar higiene de las superficies dentales, ${ }^{17}$ el de cobertura lengua planteado por Mantilla y colaboradores para determinar higiene de lengua ${ }^{18} \mathrm{y}$ una modificación del índice de 
evaluación de higiene de prótesis planteado por Pietrovsky ${ }^{19}$ para determinar la higiene de bases de prótesis y que se denominó índice de evaluación de higiene de prótesis (IEHP). Las variables relacionadas con higiene oral (ICP, IEHP y cobertura de lengua) y elementos de higiene se recolectaron antes y después de la intervención.

Se aplicó un formulario de autodiligenciamiento a los cuidadores, donde se registraron variables sociodemográficas como edad, género, procedencia e institución en la que labora y los conocimientos y percepciones de estos acerca de la higiene oral y su relación con la salud general del adulto mayor, variables que se recolectaron antes de la intervención.

Con la finalidad deevaluar el instrumento utilizado para la recolección de la información, se efectuó una prueba piloto que permitió mejorar la calidad, precisión y pertinencia de las preguntas, así como estandarizar los procedimientos de diagnóstico. La prueba piloto se realizó en cuidadores y adultos mayores pertenecientes a una institución diferente a las dos participantes en el estudio y con características semejantes a la población objeto de estudio.

Para la recolección de la información se coordinó con los directivos de las instituciones seleccionadas, informando los objetivos del estudio y solicitando el consentimiento informado de los participantes. Para la recolección de la información relacionada con condición oral en los adultos mayores, estos fueron examinados bajo condiciones estandarizadas utilizando una unidad portátil y de instrumental básico de examen. Posterior al examen oral se proporcionó elementos de higieneoral a todos los adultos mayores de las dos instituciones.

La planeación y diseño del programa se fundamentó en los resultados obtenidos de las encuestas previas tanto de cuidadores como de adultos mayores; se diseñaron dos estrategias educativas, que fueron aplicadas en forma simultánea en las dos instituciones; la asignación para ser grupo intervención y grupo control se definió por conveniencia (grupo control: Asilo San Antonio, grupo intervención: Asilo San Rafael).

Grupo control (Asilo San Antonio): Los cuidadores de este grupo se intervinieron con una estrategia tradicional, orientada a mejorar la higiene oral de los adultos mayores y que estuvo constituida por una intervención puntual constituida por una sola fase. FASE I Aporte de conocimientos: se realizó una conferencia que se presentó en Power Point, la cual incluyó condición actual del adulto mayor, aspectos básicos de higiene oral en adultos mayores e importancia del cuidador en el mejoramiento de la calidad de vida de las personas a su cargo.

Grupo intervenido (Asilo San Rafael): Intervenido con una estrategia especial diseñada por los investigadores y que constaba de: FASE I Sensibilización: se realizó mediante un póster, alusivo a la importancia del adulto mayor. FASE II Aporte de conocimientos: se realizó una conferencia igual a la aplicada en el grupo control, en la que se incluyeron los mismos contenidos teóricos y especificaciones de diseño. FASE III Motivación: utilizó como material educativo, separadores de libros recalcando la importancia de la atención de cui-dadores en el bienestar del adulto mayor. FASE IV Refuerzo de la información: se realizó un afiche cuyo propósito fue reafianzar y sintetizar los conceptos de higiene. FASE V Mecanismo de monitoreo: se diseñó un calendario, con gráficos y temas relacionados con el cuidado oral de los adultos mayores, en el cual sellevó el control delas rotaciones de cuidadores en las diferentes tareas dela institución. FASE VI Cierre del programa: el resumen del programa se integró en un plegable con el objetivo de incentivar a los cuidadores para continuar ejerciendo control sobre los factores de riesgo que influyen directamente en la higiene oral del adulto mayor bajo su cuidado.

Luego de la aplicación del programa, se realizó la evaluación de los cambios generados por éste, en el grupo intervenido y en el grupo control, mediante la evaluación de los elementos de higiene oral y la higiene oral de los adultos mayores (ICP, IEHP y cobertura de lengua).

La base de datos se elaboró en Excel ${ }^{20}$ y se exportó al paquete estadístico STATA 8.021 para su correspondiente análisis. Inicialmente, las variables se describieron según medidas de resumen según su naturaleza; adicionalmente cada una de las variables sociodemográficas, de conocimientos y percepciones de los cuidadores se analizaron según institución; deigual manera las variables sociodemográficas y clínicas recolectadas en adultos mayores se compararon según institución intervenida o no intervenida mediante test Chi Cuadrado y test exacto de Fisher. ${ }^{22}$ Se consideró un nivel de significancia $\alpha=0.05$

42 
La investigación se realizó dentro de los principios éticos establecidos en la resolución 008430 de 1993 del Ministerio de Protección Social de la República de Colombia y en los que se preservaron los principios de autonomía, beneficencia y justicia. ${ }^{23}$

\section{RESULTADOS}

\section{CUIDADORES}

La encuesta aplicada a cuidadores la respondieron el 83\% (25) deellos. El 96\% (24) delas personas encuestadas eran mujeres; el 72\% (18) estaban vinculados laboralmente al asilo San Rafael y El 76\% de los cuidadores procedían de Bucaramanga (Tabla 1).

El promedio de edad de los cuidadores fue de $35.5 \pm 9.7$ años con rangos de edad entre los 18 y 62 años.
Al considerar la procedencia, se pudo observar diferencias estadísticamente significativas por institución $(p=$ 0.032), pues mientras el $88.8 \%$ de los cuidadores de San Rafael procedían de Bucaramanga, el $42.9 \%$ de los de San Antonio procedían de esta misma ciudad (Tabla 1).

Percepción de las condiciones de salud del adulto mayor: El $64 \%$ (16) de los cuidadores encuestados consideran que algunos adultos mayores están en capacidad física de realizar su higiene oral y el 76\% (19) afirman que algunos de los adultos mayores tienen condiciones mentales adecuadas para realizar las actividades de autocuidado (Tabla 2).

La percepción que tenían los cuidadores sobre la capacidad tanto física como mental de las personas mayores hacía el autocuidado, mostraron diferencias estadísticamente significativas al analizarse según institución,

Tabla 1. Caracteristicas sociodemográficas del grupo de cuidadores descrito en forma global y según institución involucrada.

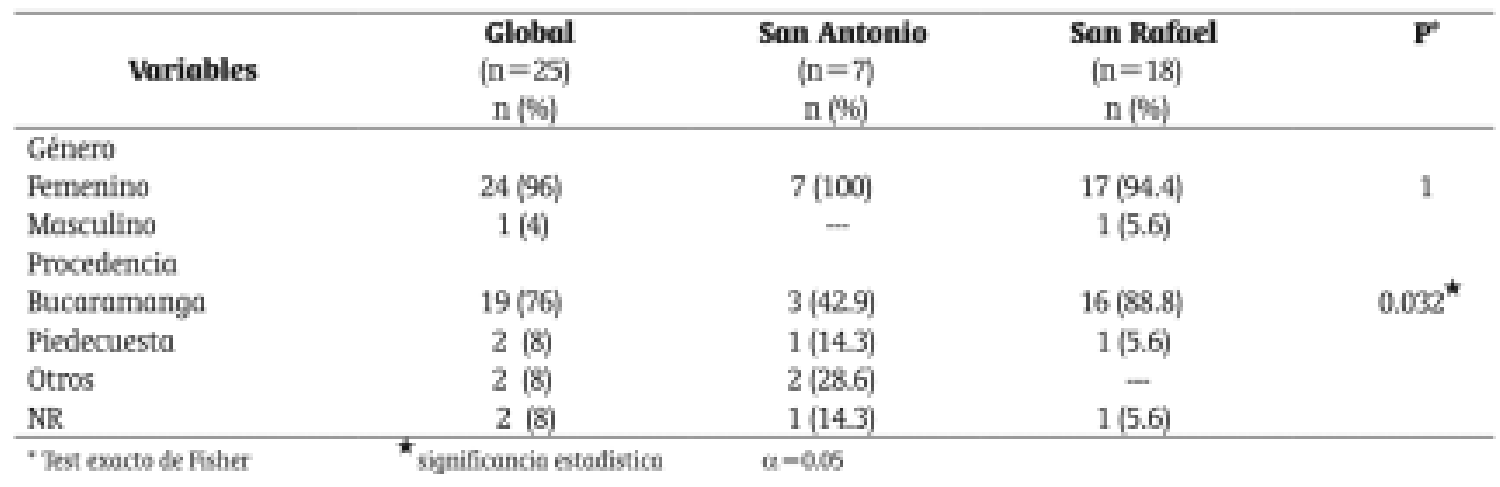

Tabla 2. Percepción que tienen los cuidadores sobre la condición fisica y mental del adulto mayor descrito en forma global y según institución en la que trabajan.

\begin{tabular}{|c|c|c|c|c|}
\hline Variable & $\begin{array}{c}\text { Clobal } \\
(n=25) \\
n(96)\end{array}$ & $\begin{array}{c}\text { San Antonio } \\
(n=7) \\
n(\%)\end{array}$ & $\begin{array}{c}\text { San Rafael } \\
(n=18) \\
n(96)\end{array}$ & $\mathbf{P}^{*}$ \\
\hline Ninguno & $1(4)$ & $1(14.3)$ & - & $0.003^{\star}$ \\
\hline Poc05 & $4(16)$ & $3(42.8)$ & $1(5,6)$ & \\
\hline \multirow[t]{2}{*}{ Algunos } & $16(64)$ & $1(14.3)$ & $15(83.3)$ & \\
\hline & 4 (16) & $2(28.6)$ & 2 (11.1) & \\
\hline Ninguno & $2(8)$ & $2(28.6)$ & - & 0.032 * \\
\hline Pocos & $2(8)$ & $1(14.3)$ & $1(5.6)$ & \\
\hline Algunes & 19 (76) & $3(42.8)$ & 16 (89) & \\
\hline Mayoria & $2(8)$ & $1(14.3)$ & $1(5.6)$ & \\
\hline
\end{tabular}


Tabla 3. Conocimientos de los cuidadores sobre salud oral y cuidado oral en las personos mayores descrito en forma global y por institución estudioda antes de la intervención.

\begin{tabular}{|c|c|c|c|c|}
\hline Variable & $\begin{array}{c}\text { Global } \\
(\mathrm{n}=25) \\
\mathrm{n}(96)\end{array}$ & $\begin{array}{c}\text { San Antonio } \\
(n=7) \\
\mathrm{n}[\%)\end{array}$ & $\begin{array}{c}\text { San Rafael } \\
(\mathrm{n}=18) \\
\mathrm{n}(36)\end{array}$ & $\mathbf{P}^{*}$ \\
\hline \multicolumn{5}{|l|}{ Elementos de higiene } \\
\hline Cepillo y crema & $25(100)$ & $7(100)$ & $18[100]$ & \\
\hline Seda & $16(64)$ & $5(71)$ & $11(61)$ & 1.0 \\
\hline Enjungue & 17 (68) & $7(100)$ & $10(56)$ & $0.057^{\star *}$ \\
\hline Palillos & $3(12)$ & $1(14.3)$ & 2 (11.1) & 1.0 \\
\hline Hilo & $1(4)$ & - & $1(5.6)$ & 1.0 \\
\hline Otros & $1(4)$ & - & $1(5.6)$ & 1.0 \\
\hline
\end{tabular}

pues mientras el 83.3\% (15) de los cuidadores de San Rafael consideraron que algunos adultos mayores a su cargo estaban en condiciones físicas adecuadas para desempeñar su cuidado oral, sólo el 14.3\% (1) delos cuidadores deSan Antonio tenían esta misma opinión. En cuanto a la salud mental, el 89\% (16) de los cuidadores de San Rafael piensan que algunos de los adultos mayores tienen una condición mental adecuada para mantener conductas de autocuidado, el $43.2 \%$ de los San Antonio tienen esta misma opinión mostrando también diferencias estadísticamente significativas (Tabla 2).

\section{Conocimientos sobre cuidado oral}

Al interrogar a los cuidadores sobre la relación de la salud oral con la condición general el 100\% (25) de los encuestados tenían claro que estaban relacionadas; de igual forma se logró observar la relación que los cuidadores estudiados establecían entre edentulismo y alteraciones nutricionales.

El 100\% delos cuidadores seleccionaron una técnica adecuada de cepillado dental y consideraron quela prótesis dental se debía cepillar por fuera de boca. Así mismo respondieron que el cepillo y la crema dental eran los elementos de higiene oral que debían usar los adultos mayores (Tabla 3).

El análisis de variables relacionadas con conocimientos sólo mostraron diferencias marginalmente significativas para la variable uso de enjuagatorio en los adultos mayores $(p=0.057)$, fue más frecuentemente considerada su uso en San Antonio por el $100 \%$ de los encuestados mientras que en San Rafael fue seleccionada por $56 \%$ de los cuidadores (Tabla 3).

\section{ADULTOS MAYORES}

\section{Antes de la implementación del programa}

\section{Variables sociodemográficas}

Se evaluaron 91 adultos mayores, 47 (51.6\%) estaban vinculados al Asilo San Antonio, 45 (51.6\%) eran mujeres, 52 (57\%) reportaban tener algún nivel deeducación, 53 (59\%) procedían de zona rural; 44 (48.4\%) consideraban quesu situación económica era mala y 82 (90.1\%) estaban vinculados al pabellón de caridad (Tabla 4).

Es importante resaltar que el promedio de edad en la población adulta mayor evaluada fuede $75.9+10.5$ años, la media de años de educación fue de $1.9+3.02$ años y con una mediana de 1 año.

Las variables que mostraron diferencias estadísticamente significativas según tipo de institución fueron género $(p<0.001)$ y procedencia $(p=0.016)$; para sexo esto era de esperar ya que una sólo al berga mujeres (San Antonio); mientras quela otra sólo hombres (San Rafael).

En cuanto a procedencia se destaca que mientras en San Antonio el 25.5\% (12) de los encuestados decían que venían de un pueblo, en San Rafael sólo el 4.5\% (2) hicieron esta misma afirmación (Tabla 4).

La edad, ni los años de educación, mostraron diferencias estadísticamente significativas al compararlas por institución con niveles $p=0.9233$ y $p=0.2217$, respectivamente.

\section{Elementos de higiene oral}

El $74.7 \%(68)$ y $75.8 \%$ (69) de las personas mayores eva- 
Tabla 4. Descripción de las caracteristicas sociodemográficas en forma global y según institución de la población adulta mayor institucionalizada.

\begin{tabular}{|c|c|c|c|c|}
\hline Variable & $\begin{array}{c}\text { Global } \\
(\mathrm{n}=91) \\
\mathrm{n}[\%]\end{array}$ & $\begin{array}{c}\text { San Antonio } \\
(\mathrm{n}=\mathbf{4 7 )} \\
\mathrm{n}(\%)\end{array}$ & $\begin{array}{c}\text { San Rafael } \\
\begin{array}{c}(n=44) \\
n\end{array}(\%)\end{array}$ & $\mathbf{P}^{*}$ \\
\hline \multicolumn{5}{|l|}{ Género } \\
\hline Femenino & $47[51.5)$ & $47(100)$ & - & $<0.0011^{\star}$ \\
\hline Mosculino & $44(48.5)$ & - & $44(100)$ & \\
\hline \multicolumn{5}{|l|}{ Educación } \\
\hline Algún nivel & $52[57.1)$ & $30[63.8]$ & $22(50)$ & $0.183^{*+4}$ \\
\hline Ningún nivel & $39(42.9)$ & $17(36.2)$ & $22(50)$ & \\
\hline \multicolumn{5}{|l|}{ Procedencia } \\
\hline Compo & 535827 & $23[48.9]$ & 30 (68.2) & $0.016^{\star}$ \\
\hline Pueblo & $14(15.4)$ & 12 [25.5) & 2 (4.5) & \\
\hline Ciudød & $24(26.4)$ & 12 [25.5) & $12(27-3)$ & \\
\hline \multicolumn{5}{|c|}{ situación económica } \\
\hline Mola & $44(48.4)$ & $27(57.4)$ & 17 [38.6] & $0.196^{+4}$ \\
\hline Regular & $29[31.9]$ & 12 (25.5) & 17 (38.6) & \\
\hline Buena & $18(19.8)$ & $8(17)$ & $10(227)$ & \\
\hline \multicolumn{5}{|l|}{ Pabeilón } \\
\hline Coridad & $82[90.1)$ & $44(93.6)$ & 38 (86.4) & 0.306 \\
\hline Pensionados & $9(9.9]$ & $3(6.3)$ & $6(13.6)$ & \\
\hline \multicolumn{5}{|l|}{ Trabaja } \\
\hline $\mathrm{Si}$ & $61[67]$ & $32[68.1]$ & $29[65.9]$ & $0.825^{+4}$ \\
\hline No & $30(33)$ & $15[31.9]$ & 15[34.1] & \\
\hline
\end{tabular}

Tabla 5. Descripción global y por institución de las variables relacionadas con higiene oral antes de la intervención.

\begin{tabular}{|c|c|c|c|c|}
\hline Variable & $\begin{array}{c}\text { Global } \\
\mathbf{n}=91 \\
\mathbf{n}(\%)\end{array}$ & $\begin{array}{c}\text { San Antonio } \\
n=47 \\
n(\%)\end{array}$ & $\begin{array}{c}\text { San Rafael } \\
n=44 \\
n\left(\%_{0}\right)\end{array}$ & $\mathbf{P}^{*}$ \\
\hline Bueno & $16(17.5)$ & $7(14.9)$ & 9 [20.5] & $0.869^{4+}$ \\
\hline Regular & $32(35.2)$ & $18(38.3)$ & $14(31.8)$ & \\
\hline Mala & $28(30.8)$ & $14(29.8)$ & $14(31.8)$ & \\
\hline No aplica & 15 (16.5) & $8(17)$ & 7 [15.9] & \\
\hline Bueno & $5(5.5)$ & $2(4.3)$ & $3(6.8)$ & 0.271 \\
\hline Regular & $12(13.2)$ & $4(8.5)$ & 8 (18.2) & \\
\hline Mala & $44(48.4)$ & $27(57.4)$ & $17[38.6]$ & \\
\hline No aplica & 30 (32.9) & $14(29.8)$ & $16(36.4)$ & \\
\hline Sin & $2(2.2)$ & $2(4.3)$ & - & 0.322 \\
\hline Delgoda & $9(9.9)$ & $6(12,8)$ & $3(6.8)$ & \\
\hline Gruesa & $79(86.8)$ & $39(83)$ & 40 [90.9] & \\
\hline No aplica & $1(1.1)$ & - & $1(2.3)$ & \\
\hline
\end{tabular}

luadas afirmaron utilizar el cepillo y la crema dental; el agua y el jabón fueron utilizados por el $6 \%$ de los sujetos. Otros elementos como la seda registraron una baja frecuencia de uso que no superó el $2 \%$. No se observaron diferencias estadísticamente significativas para estas condiciones según institución.

\section{Nivel de higiene dental, prótesis y lengua}

Veintiocho (35.2\%) personas mayores registraban regular y $28(30.8 \%)$ mala higiene de las superficies dentales. Cuarenta y cuatro $(48.4 \%)$ presentaban mala higiene de las prótesis dentales y 30 (32.9\%) no fue posible evaluar este criterio por no tenerlas al momento del examen. En 
Tabla 6. Descripción global y por institución de las variables relacionadas con higiene oral después de la intervención.

\begin{tabular}{|c|c|c|c|c|}
\hline Variable & $\begin{array}{c}\text { Global } \\
\mathbf{n}=91 \\
\mathbf{n}(\%)\end{array}$ & $\begin{array}{c}\text { San Antonio } \\
n=47 \\
n(\%)\end{array}$ & $\begin{array}{c}\text { San Rafael } \\
n=44 \\
n\left(W_{0}\right)\end{array}$ & $\mathbf{P}^{\phi}$ \\
\hline \multicolumn{5}{|l|}{ ICP } \\
\hline Bueno & $27(29.6)$ & $7(14.9)$ & $20(45.5)$ & $0.005^{\star}$ \\
\hline Regular & $31(34.1)$ & $18(38.3)$ & $13(29,6)$ & \\
\hline Mala & $18(19.8)$ & $14(29.8)$ & $4[9.1]$ & \\
\hline No aplica & $15(16.5)$ & $8(17)$ & $7(15.9)$ & \\
\hline \multicolumn{5}{|l|}{ IEHP } \\
\hline Bueno & $15(16.5)$ & $3(6.4)$ & $12(27.3)$ & $0.002^{\star}$ \\
\hline Regular & $9(9.9)$ & $3(6.4)$ & $6(13.6)$ & \\
\hline Mala & $37(40.7)$ & $27(57.4)$ & $10(22.7)$ & \\
\hline No aplica & $30(32.9)$ & $14(29.8)$ & $16(36.4)$ & \\
\hline \multicolumn{5}{|l|}{ Cobertura lengua } \\
\hline $\operatorname{Sin}$ & $4(4.4)$ & $1(2.1)$ & $3[6.8]$ & 0.485 \\
\hline Delgada & $21(23.1)$ & $12(25.5)$ & $9(20.5)$ & \\
\hline Gruesa & $65(71.4)$ & $34(72.3)$ & $31(70.4)$ & \\
\hline No aplica & $1(1.1)$ & - & $1[2.3]$ & \\
\hline
\end{tabular}

cuanto a cobertura de lengua 79 (86.8\%) adultos mayores registraban una cobertura gruesa de la lengua (Tabla 5).

No se observaron diferencias estadísticamente significativas en aspectos relacionados con la higiene dental, de superficie de prótesis dentales y de cobertura del lengua al analizar estos criterios según institución antes de la intervención (Tabla 5).

\section{Después de la implementación del programa}

\section{Elementos de higiene oral}

Al analizar este criterio se observa que los elementos como el cepillo y la crema son usados por el $82.4 \%$ (75) de los sujetos y son empleados por igual en ambas instituciones; el agua y el jabón son usados por el 3.3\% (3) delos adultos mayores encuestados y la seda por el $1.1 \%$ (1) de los sujetos.

\section{Nivel de higiene dental, prótesis y lengua}

Treinta y un (34.1\%) y 27 (29.6\%) personas mayores registran regular y buena higiene de superficies dentales; ${ }^{37}$ $(40.7 \%)$ y 30 (32.9\%) de los adultos evaluados registran mala higiene de prótesis dentales o no podían ser evaluados y finalmente $65(71.4 \%)$ de las personas registraban una cobertura gruesa de la lengua (Tabla 6).

Al analizar según institución intervenida se observó que el $14.9 \%$ (7) de los adultos mayores del asilo San Antonio (grupo control) registraba buena higiene dental mientras que el $45.5 \%$ (20\%) de los mayores del asilo San Rafael (grupo intervención) registraban esta misma condición, lo que muestra diferencias estadísticamente significativas $(p=0.005)$.

Al analizar higiene de prótesis dentales también se observaron diferencias estadísticamente significativas $(p=0.002)$ entre San Antonio y San Rafael pues mientras en la primera el $6.4 \%$ (3) de los personas evaluadas registraba buena higiene de prótesis el 27.3\% (12) de San Rafael registraba esta misma condición. Sin embargo, al analizar cobertura de lengua no se observó diferencias entre San Antonio y San Rafael después de la intervención $(p=0.485)$.

\section{DISCUSIÓN}

En la literatura revisada se han encontrado pocos reportes de programas educativos en salud oral de adultos mayores institucionalizados, dirigido a sus cuidadores, y ninguno que fuese aplicable a las condiciones actuales de los centros geriátricos de la región; pues en éstos, el tipo de personal que integra el grupo de cuidadores, en la mayoría de los casos, no han alcanzado una formación técnica.

Nicol y colaboradores reportaron sobre un programa de entrenamiento en salud oral dirigido a los trabajadores

\section{6}


de cuidado en salud en hogares geriátricos integrado fundamental por personal técnico y especializado,; ${ }^{24}$ sin embargo, a pesar de las diferencias en la formación académica los resultados en las dos investigaciones mostraron resultados satisfactorios y con impacto sobre el mejoramiento en los indicadores de salud e higiene oral en los adultos mayores.

Se determinó desarrollar un programa educativo, el cual inició identificando el nivel de higiene oral de las personas institucionalizadas y los conocimientos y percepciones de sus cuidadores, tal como lo plantea Nicol y colaboradores, y Kenneth y colaboradores como requisito elemental para la realización de este tipo de programas. ${ }^{2425}$

Los resultados que arrojaron las encuestas preliminares del estudio, indican el alto nivel de conocimientos de higiene oral de los cuidadores; resultados que contradicen a los del estudio que antecedió al presente, realizado por Díaz y colaboradores, que indicó que los cuidadores de estas dos instituciones no contaban con los conocimientos suficientes sobre el cuidado de adultos mayores, ${ }^{15}$ así mismo, Preston y colaboradores al igual que Wardh y colaboradores describieron quelos conocimientos de los profesionales de la salud, respecto a las condiciones orales del adulto mayor, son deficientes en algunas instancias. ${ }^{26,27}$

Al realizar el examen clínico de los adultos mayores, se observó un alto nivel de mala higiene oral, similar al encontrado en los estudios de Bermúdez y colaboradores, y Espíndola y colaboradores quefueron realizados en un grupo significativo de instituciones geriátricas de Bucaramanga y su área metropolitana. ${ }^{28,29}$

Para la recolección de la información sobre el nivel de higiene oral de los adultos mayores se utilizó el índice de cobertura de lengua, con el propósito de lograr un examen clínico de higiene oral más completo debido a que esta estructura ha sido olvidada posiblemente por la necesidad de concentrarseen la protección y tratamiento de los tejidos dentales duros y estructuras de soporte; y cuando ésta ha sido incluida en estudios, como los realizados por Mantilla y colaboradores, Moreno y colaboradores, es utilizada sólo, para evaluar los microorganismos presentes en ella o como agente causal de halitosis. ${ }^{16-30}$

Luego de la aplicación del programa en el grupo intervenido se observaron cambios estadísticamente significa- tivos en higiene dental; aumentó $25 \%$, la higiene oral buena. En cuanto a buena higiene de prótesis, aumentó aproximadamente en un 20\%. En higiene de lengua, se observó, aumento de casi un $7 \%$ en lengua sin cobertura pero no alcanzó a mostrar diferencias estadísticamente significativas. Esto último se debió probablemente al reporte de sensibilidad frente al reflejo nauseoso manifestado por un número considerable de adultos mayores al momento del examen clínico, además, por la forma de obtención de los resultados de cobertura, arrojada por el índice utilizado en el estudio, el cual no permitió observar objetivamente en los cambios que se puedan originar respecto a la higiene de lengua.

Con el programa educativo se evidenciaron cambios en la higiene oral de los adultos mayores sólo después de realizada la intervención; reafirmando lo mencionado por Rakowski y colaboradores quienes sugieren que no es suficiente con poseer los conocimientos básicos adecuados, sino que además es importante reforzar, motivar y sensibilizar a los cuidadores para que los apliquen y difundan a las personas que tienen a cargo. ${ }^{31}$

Los resultados obtenidos con la intervención demuestran que la implementación de programas educativos en cuidadores, son una buena estrategia comunicativa y preventiva para lograr un impacto a corto plazo, en la higiene oral de adultos mayores y coincide con lo expresado por Nicol y colaboradores. ${ }^{24}$

Por lo anterior se hace necesario, según lo establecido por los investigadores y reiterado por Rakowski y colaboradores, en su estudio, incentivar constantemente a los cuidadores para garantizar mayor efectividad y continuidad de dichos programas, en pro del mejoramiento no sólo de la higiene oral del adulto mayor sino de su calidad de vida. ${ }^{31}$

Se podría concluir que la implementación de un programa en salud oral dirigido a los cuidadores de las instituciones geriátricas San Antonio y San Rafael, mostró que acciones puntuales no tiene la misma eficacia que un conjunto de intervenciones continuas, encaminadas en un programa educativo y comunicativo.

\section{Agradecimientos}

A la comunidad integrante de los asilos San Antonio y San Rafael. 


\section{BIBLIOGRAFÍA}

1. Aristizabal N, Ortiz L. Cuidadores formales mayores de sesenta años, pertenecientes a cuatro centros geriátricos de la ciudad de Bogotá y a tres del municipio de Chía y la salud mental: depresión. Asociación Colombiana de Gerontología y Geriatría 2002; 6.

2. Baldón M et al. El envejecimiento de la cavidad oral y de sus tejidos. Odontogeriatría. 3495 - 3499.

3. Fuentes P. Conocimiento integral del paciente. Odontogeriatría. 21: $6-9$.

4. Banting DW. Epidemiology of root caries. Gerontology 1986.

5. Athena S. Relationship of diet to root caries. En: the American journals of clinical nutrition 1995; 61: 423 -.

6. Lin HC. Coronal and root caries in southern Chinese adults. J Dent Res 2001; 80: 1455 - 1480.

7. Botero BE. El cuidado integral la familia y el cuidador. Asociación Colombiana de Gerontología y Geriatría 2000; 14.

8. Sheiham A, SteeleJG. Therelationship among dental status nutrient in take and nutritional status in older people. J Dent Res 2001; 80: $408-413$.

9. Dormenvar V. Association between malnutrition, poor general health and oral dryness in hospitalized elderly patients. Aged and Ageing 1998; 27: 123 - 128.

10. Santillana SP. Depresión en la población adulta mayor: Tamizaje en unidad de primer nivel de atención médica. Revista Médica 1999; 37: 111 - 115.

11. Marín DJ. Cavidad oral y nutrición en el adulto mayor. Asociación Colombiana de Gerontología y Geriatría 1999; 13: -

12. Grando TV, Mehr D. Why older adults with light care needs enter and remain in nursing homes. J of Gerontological nursing 2002; 28:

13. Cruz GD. El viejo deayer a hoy variaciones de su rol socio familiar. Revista de la Asociación Colombiana de Gerontología y Geriátrica 2003; 17: 462 - 468.

14. Vigil M. Dental caries and need for treatment among institutionalized elderly. Comm Dent Oral Epidemiol 1989; 17: 102 - 105.

15. Díaz M, Pinto D, Mejia LY, González X, Miranda Cv. Conocimientos, actitudes y prácticas en higiene oral de los cuidadores de adultos mayores residentes en el asilo San Rafael y San Antonio de la cuidad deBucaramanga.

16. CDC-OMS. Epi-Inf 6 Versión 6.04a Noviembre de 1996.

17. Pieschacón MP, Camargo DM. Acuerdo entre el índice de Green y Vermillion con el índice cuantificado de placa. Ponencia XI Encuentro Nacional de Investigación Odontología. CES. Medellín Septiembre 2000.
18. Mantilla S, Danser MM, Sipos P, Row ani B, Van der Velden U, Van der Wijden F. Cobertura de la lengua y cuentas bacterianas salivares en sujetos sanos y con gingivitis y en pacientes con periodontitis. Ustasalud 2002; 1: 7 - 17.

19. Pietrokovski. Oral findings elderly nursing home residents in selected counties. Oral hygiene conditions and plaque accumulation on dentadure surfaces. J Prosthod Dent 1995; 73: 136 -141.

20. Microsoft Excel, 5.0, Microsoft Corporation, 1997.

21. Statacorp 2003. Stata Statistical Software: Release 8.0. College Station, TX:Stata Corporation.

22. Pagano M, Gauvreau K. Principles of Bioestatistics. Duxbury press, Belmont, 1993.

23. República de Colombia. Ministerio de Salud. Resolución No. 008430 del 4 de octubre de 1993.

24. Nicol R, Sw eeney Mp, Mchugh S, Gagg J. Effectiveness of health carew orker training on theoral health of elderly residents of nursing homes. Commununity Oral Dent Epidemiol 2005; 115 - 124.

25. Kenneth A, Freedman. Odontología preventiva, cuidado de la salud bucal en el paciente geriátrico. Ed. Mundi S.A.; 1981. p. 193 204.

26. Preston AJ, Punekar S. Oral care of elderly patients: nurses knowledge and views. the fellow ships of medicine 2000; 76: 89 - 91.

27. Wardh I, Lillemor R, Hall berg M, Berggren U, Anderson L, Sorensen S. Oral Health - A low priority in nursing. ScandJ Caring Sci 2000; 14: $138-142$.

28. Bermúdez W, Concha SC, Camargo DM. Perfil orofacial de las personas mayores institucionalizadas de la ciudad de Bucaramanga y su área metropolitana. Ustasalud Odontología 2003; 2: 13 - 19.

29. Espíndola G, Pinzón A. Concha SC, Camargo DM. Asociación entre el estado mental y la condición oral de la persona adulta mayor institucionalizada de Bucaramanga y su área metropolitana. Ustasalud Odontología 2003; 2: 21 - 32.

30. Rodríguez MI, Moreno L, Martínez CA. Factores orales asociados a halitosis en pacientes usuarios de prótesis total que asisten a las clínicas odontológicas de la Universidad Santo Tomás. Ustasalud Odontología 2005; 4: 9 - 16.

31. Rakow ski W, Lang WP, Kerschbaum WE. Correlates of interest in dental health education with older adults: Future perspective and quality of clinical interaction. Gerodontics 1987; 3: 191 - 197.

\section{8}

Ustasalud Odontología 2006; 5: 40 - 48 García Y. y col. 